\title{
Dural effects of oxidative stress on cardiomyogenesis via Gata4 transcription and protein ubiquitination
}

\author{
Tao Li', Xia Zhang ${ }^{1}$, Kesheng Jiang ${ }^{2}$, Jing Liư ${ }^{3}$ and Zhiqiang Liu $\mathbb{C}^{3}$
}

\begin{abstract}
Oxidative stress generates reactive oxygen species (ROS) that can promote or inhibit cardiac differentiation of stem cells dependent on the intensity of stimuli as well as cellular context in redox and differentiation status. In the current study, we confirmed that suitable intensity of hydrogen peroxide at the formation stage of embryoid bodies (EBs) effectively favored the formation of spontaneously beating cardiomyocytes from P19 embryonal carcinoma cells. Mechanistic studies implicated that extrinsic ROS enhanced the Caspase-mediated degradation of Oct4 and Nanog, two factors that governing pluripotent property. Further experiments suggested that a cohort of Nanog together with histone deacetylase 4 (Hdac4) played a critical role in establishing and maintaining the silent transcriptional status of Gata4 and Nkx2.5 in undifferentiated cells. Thus, an impulse of hydrogen peroxide depleted Nanog and Hdac4 via a caspase-dependent manner to ameliorate the repression on Gata4 and Nkx2.5 promoters, thereby generating a persistent activation on cardiac differentiation program. Meanwhile, we found that excessive ROS-activated JNK cascade to facilitate the ubiquitination and subsequent degradation of Gata4 protein. Overall, our results indicate that suitable ROS promotes the activation of Gata4 in transcription, while excessive ROS targets Gata4 protein for proteasome-dependent degradation. Gata4 is an important modulator balancing the promoting and inhibitory effects of oxidative stress on differentiation program of cardiomyogenesis.
\end{abstract}

\begin{abstract}
Introduction
Cardiac differentiation is a complicated stepwise process, which is tightly regulated by the chronological integration of transcriptional program and signaling pathways. The precise role of distinct signaling pathway during cardiomyogenesis is dependent on the cell context and the differentiation stage, exhibiting remarkable stagespecific and dose-dependent characteristics. Several signaling pathways have been revealed to be associated with the stepwise process of cardiac differentiation, such as
\end{abstract}

Correspondence: Tao Li (litao7997@hunnu.edu.cn) or Zhiqiang Liu (zhiqiangliu@tmu.edu.cn)

${ }^{1}$ School of Medicine, Hunan Normal University, Changsha, Hunan 410013, China

Department of Biology, Zhejiang Normal University, Jinhua, Zhejiang 321004, China

Full list of author information is available at the end of the article Edited by A. Stephanou
TGF- $\beta$, Wnt, and Notch ${ }^{1}$. These signaling pathways exert distinct effect and govern the determination of cell fate during the critical steps of cardiac differentiation. Manipulating signaling pathways can direct the committed differentiation of stem cells to the cardiac lineages, thereby efficiently yielding homogeneous and sufficient number of cardiomyocytes ${ }^{2,3}$.

Oxidative stress stimulates the production of reactive oxygen species (ROS), a group of highly reactive molecules, which participate in various biological events as an intracellular signal. However, the excessive emergence of ROS also causes cell damage or disorder. Thus, oxidative stress can be beneficial or deleterious, depending on the magnitude and duration of stimuli, and the overall redox context and differentiation status of cells. In adult heart, ischemic injury produces massive ROS, which predisposes cardiomyocytes to chronic dysfunction, damage, and 
death. On the other hand, the burst of ROS also activates the proliferation and differentiation of endogenous or implanted stem cells to repair the damaged myocardium. Importantly, differentiation of embryonic stem (ES) cells towards cardiomyocytes can be elevated by ROS generated by intracellular NADPH oxidases or from extrinsic supplement at the formation stage of embryoid bodies $(\mathrm{EBs})^{4-7}$. Conversely, free radical scavengers can exert opposite effects on cardiac differentiation of stem cells. However, the precise mechanisms of ROS and downstream signaling transduction in cardiac differentiation remain to be elucidated. Specially, the rationale for a burst of ROS stimulation to modify cardiac gene program is mainly unknown.

P19 embryonic carcinoma cells are multi-potent and can differentiate into derivatives of all three germ layers. A combination of EB formation and DMSO induction can efficiently drive P19 cells to differentiate into cardiac myocytes. In this study, we aim to study the effects of stress-producing stimuli on cardiac differentiation in P19 cells, evaluate whether stress-producing stimuli can promote or impair cardiac differentiation dependent on its intensity and duration, and explore the underlying molecular mechanisms associated with cardiac gene program and epigenetic regulation.

\section{Results}

Oxidative stress favors cardiomyogenesis of P19 cells

P19 cells were firstly incubated with DMSO in suspension, allowing cells to aggregate and form EBs, subsequently, EBs were plated for adherent growth with DMSO for another 4 days. During the late stage of cardiac differentiation, stem cells became capable of expressing cardiac contractile protein genes $\alpha-M h c$ and $\beta-M h c$ (Fig. 1a). Spontaneously contracting cardiomyocytes initially emerged from day $8-10$ and progressively expanded in number. In protein levels, Gata4 and Nkx2.5 proteins were first detected from day 4 or 6 of cardiac induction. Cardiac myosin heavy chain MF-20 expression initially appeared at day 8 and gradually increased over the following periods (Fig. 1b). Transcriptional factors, Oct4 and Nanog maintaining the pluripotent stem cell phenotype, declined from the early stage of differentiation and rapidly vanished at the late stage (Fig. 1b). Another pluripotent factor Sox2 displayed different degradation manner from Oct4 and Nanog.

As several studies revealed that inducible production of ROS at the EB formation stage can promote cardiac differentiation $^{8,9}$. Subsequently, we treated P19 cells at day 4 with different stimuli, such as $\mathrm{H}_{2} \mathrm{O}_{2}$, autophagy inducer rapamycin (Rapa), endoplasmic reticulum (ER) stress inducer tunicamycin (TM) at suitable concentrations without significant toxic effects on cell growth and survival. Interestingly, all three stimuli favored cardiomyogenesis of P19 cells, as characterized by increasing amount of beating EBs (Fig. 1c). Comparatively, treatment at the early-differentiation stage can achieve more prominent effects than at the late stage (data not shown). All three stimuli were reported to evoke oxidative stress at suitable concentrations ${ }^{10,11}$. And the differentiation-promoting effects of all three stimuli could be abolished by the pretreatment of a free radical scavenger, catalase (Fig. 1c). Excessive $\mathrm{H}_{2} \mathrm{O}_{2}$ was detrimental to the generation of spontaneously contractile cardiomyocytes (Fig. 1d). Obviously, a short-time occurrence of exotic stress at the early-differentiation stage can trigger a permanent alteration in differentiation program of cardiac induction, thereby facilitating the eventual generation of contracting cardiomyocytes.

\section{Oxidative stress activates $\mathrm{p} 38$ and JNK signaling}

Next, we investigated the temporal activation of p38 MAPK and JNK signaling. As shown in Fig. 2a, the activation of JNK and p38 MAPK during the differentiation procedure shared a similar activation pattern with biphasic kinetics. High levels of phosphorylated JNK and p38 mainly appeared and maintained at the late stage of differentiation. The complementary effect of $\mathrm{H}_{2} \mathrm{O}_{2}$, Rapa, and TM directly elevated the phosphorylation levels of p38 and JNK kinases (Fig. 2b). Further experiments validated that the activation of p38 and JNK kinases was required for efficiency differentiation. The supplement of specific inhibitors for p38 (SB203580) or JNK (SP600125) at day 4 of cardiac induction all impeded the differentiation efficiency, as evaluated by calculating the percentage of beating EBs at day 10 of induction (Fig. 2c). Meanwhile, treatment with SB203580 or SP600125 at the early stage greatly interrupted $\mathrm{H}_{2} \mathrm{O}_{2}$-enhanced cardiomyogenesis, as evaluated by calculating the percentage of beating EBs (Fig. 2c), by immunofluorescence for sarcomeric protein $\alpha$-actinin (Fig. 2d), or by flow cytometry for FITC-labeled $\alpha$-actinin staining (Fig. 2e). Thus, our results implicated that oxidative stress activates p38 MAPK and JNK signaling to promote cardiomyogenesis.

\section{Oxidative stress alters the expression of pluripotent and cardiac-specific genes}

P19 cells were treated with increasing amounts of $\mathrm{H}_{2} \mathrm{O}_{2}$ at day 4 of cardiac induction, and then were subjected for real-time PCR and immunoblotting assay. As shown in Fig. $3 \mathrm{a}, \mathrm{H}_{2} \mathrm{O}_{2}$ treatment during the early stage significantly decreased the expression of Oct 4 and Nanog, which is a feature of cell exit from the pluripotent status. As the relative expression of Oct4 and Nanog messenger RNA (mRNAs) remained stable or became slightly upregulated, the effects of $\mathrm{H}_{2} \mathrm{O}_{2}$ treatment might attribute to accelerated degradation of Oct4 and Nanog (Fig. 3b). By comparison, $\mathrm{H}_{2} \mathrm{O}_{2}$ treatment indeed enhanced the 


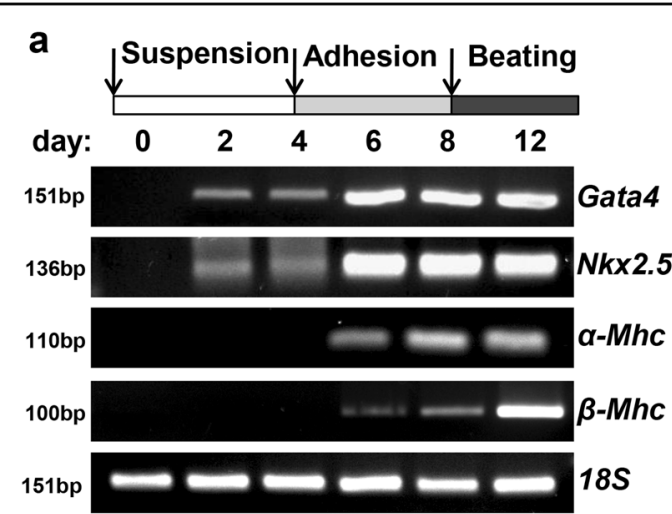

C

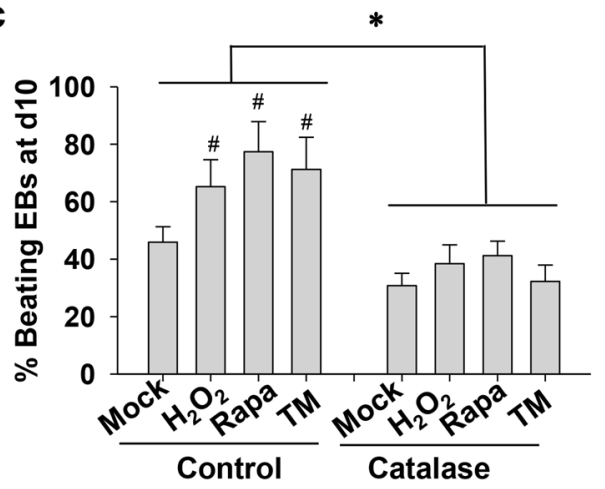

b

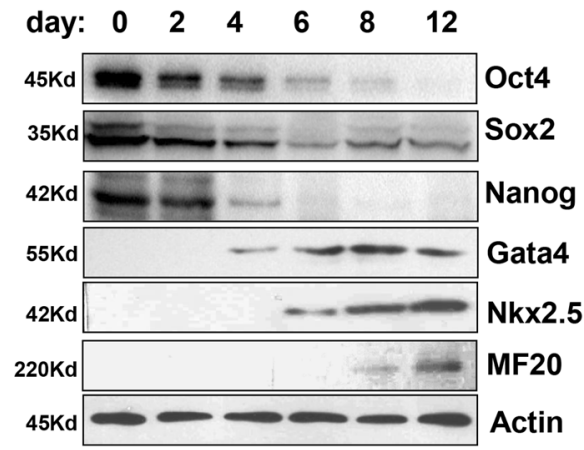

d

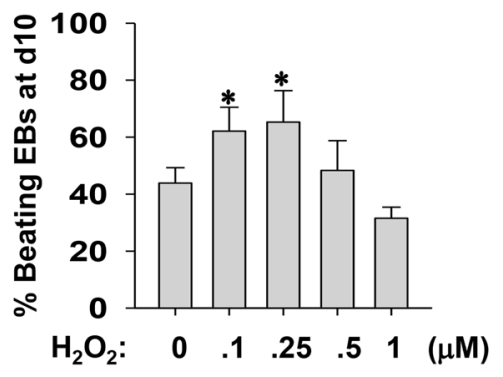

Fig. 1 Positive effects of three stress-producing stimuli on cardiac differentiation of P19 cells. a Schematic diagram of differentiation protocol with DMSO. P19 cells were cultured in suspension for embryoid body (EB) formation and then were plated for another 4 days in the presence of $1 \%$ DMSO. Cells were harvested at indicated days, and extracts were analyzed by RT-PCR to show the expression profile of cardiac transcriptional factors (Gata4 and Nkx2.5) and contractile myosin heavy chain ( $\alpha-M h c$ and $\beta$-Mhc). 185 rRNA expression was used as an internal control. b Representative western blot analysis shows the expression kinetics of pluripotent genes and cardiac-specific genes in protein level during the process of cardiac induction. c Stimulation of cardiomyogenesis by three stress-producing stimuli. P19-cell-derived EBs were treated at day 4 of cardiac induction in suspension with $100 \mathrm{nM} \mathrm{H}_{2} \mathrm{O}_{2}, 20 \mathrm{nM}$ Rapa, or $0.5 \mu \mathrm{g} / \mathrm{ml} \mathrm{TM}$. After $24 \mathrm{~h}$, the medium was changed and EBs were plated for adhesion induction. The percentage of contracting EBs was counted at day 10 among these groups. The pretreatment of ROS scavenger catalase ( $200 \mathrm{U} / \mathrm{ml}$, Sigma) for $0.5 \mathrm{~h}$ at day 4 destroyed the differentiation-promoting effects of $\mathrm{H}_{2} \mathrm{O}_{2}$, Rapa, and TM. Data obtained from three independent experiments were shown as means \pm SD. $\mathbf{d}$ The dosage-dependent effects of $\mathrm{H}_{2} \mathrm{O}_{2}$ treatment $(0,100,250,500,1000 \mathrm{nM}$ at day 4) on cardiac differentiation of P19 cells.

Differentiation efficiency of cardiomyogenesis was evaluated as the percentage of EBs with beating areas. Values are expressed as means of three independent experiments $\pm \mathrm{SD}\left({ }^{*} p<0.05 ; \# p<0.01\right.$ vs. the control)

amounts of Gata4 and $N k x 2.5$ transcripts (Fig. 3c). SB203580 and SP600125 partially reversed the $\mathrm{H}_{2} \mathrm{O}_{2}$ induced decrease of Oct 4 and Nanog in protein levels. And SP600125 was more effective in rescuing Nanog expression than SB203580 (Fig. 3d). However, high concentrations of $\mathrm{H}_{2} \mathrm{O}_{2}$ were invalid to enhance the protein amount of Gata4, although there were accompanied by an augmented Gata4 mRNA expression (Fig. 3a, b). Blockage of p38 and JNK cascades completely abolished the upregulation of Gata4 and Nkx2.5 expression (Fig. 3e).

\section{Oxidative stress activates caspase- 3 to deplete Oct4 and Nanog}

To determine the precise pathway mediating the degradation of Oct4 and Nanog, P19 cells in the early differentiation were treated with $\mathrm{H}_{2} \mathrm{O}_{2}$ at the presence of the proteasome inhibitor MG-132 and the pan Caspase inhibitor Z-VAD-FMK. As shown in Fig. 4a, Z-VAD-FMK rather than MG-132 was capable of rescuing the $\mathrm{H}_{2} \mathrm{O}_{2}$ decreased Oct 4 and Nanog. Therefore, caspase-mediating depletion accounted for the degradation of Oct4 and Nanog. In fact, we found that there existed a temporal activation of caspase- 3 at the early stage throughout the whole process of cardiac induction (Fig. 4b). The administration of $\mathrm{H}_{2} \mathrm{O}_{2}$, Rapa, and $\mathrm{TM}$ induced an intensified generation of cleaved caspase-3 (Fig. 4c). In addition, the induced activation of caspase- 3 could be reversed by the blockade of JNK cascade (Fig. 4d).

\section{Nanog degradation relieves the repression of Gata4 and Nkx2.5 transcription}

Since Z-VAD-FMK blocked the degradation of Oct4 and Nanog, we subsequently investigated the effects of caspase inhibition on Gata4 and Nkx2.5 expression. As 


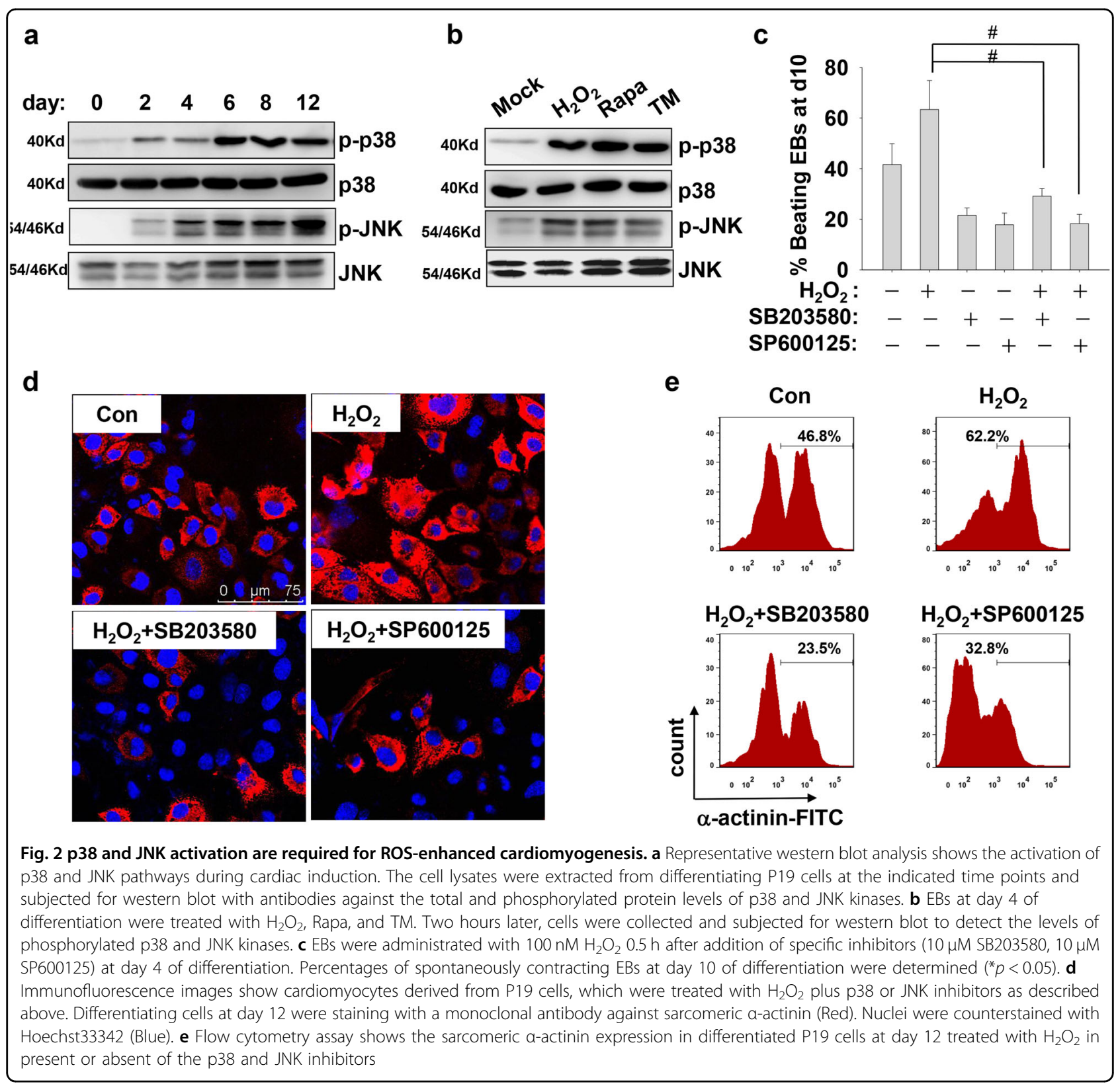

shown in Fig. 5a, Z-VAD-FMK downregulated the expression of Gata4 and Nkx2.5 mRNA at day 4 of differentiation. We further investigated whether the augment of Gata4 and $N k x 2.5$ expression in response to oxidative stress was not a result from Oct4 and Nanog degradation. Unexpectedly, ectopic expression of Oct4 in undifferentiated P19 cells effectively increased the transcripts of Gata4 and Nkx2.5 genes (Fig. 5b). Importantly, Nanog overexpression repressed Gata4 and Nkx2.5 expression. There exist multiple Nanog-binding TAATrich domains on Gata4 and Nkx2.5 promoters (Fig. 5c). ChIP assays were conducted with anti-Nanog antibody, and DNA fragments containing the putative Nanog- binding sites were detected. Along with the effect of $\mathrm{H}_{2} \mathrm{O}_{2}$ treatment on Nanog expression, the enrichment of Nanog on the promoters of Gata4 and $N k x 2.5$ genes also decreased after $\mathrm{H}_{2} \mathrm{O}_{2}$ exposure (Fig. $5 \mathrm{~d}$ ). Therefore, our results established a link between Nanog degradation and the transactivation of Gata4 and $N k x 2.5$ genes.

\section{Oxidative stress promotes Hdac4 degradation to facilitate} the expression of Gata4 and Nkx2.5

We further exploit whether other substrates of caspase3 participate in the early-stage differentiation. It is well known that histone deacetylase Hdac4 is a substrate for caspase- $3^{12,13}$. In this study, we examined whether 


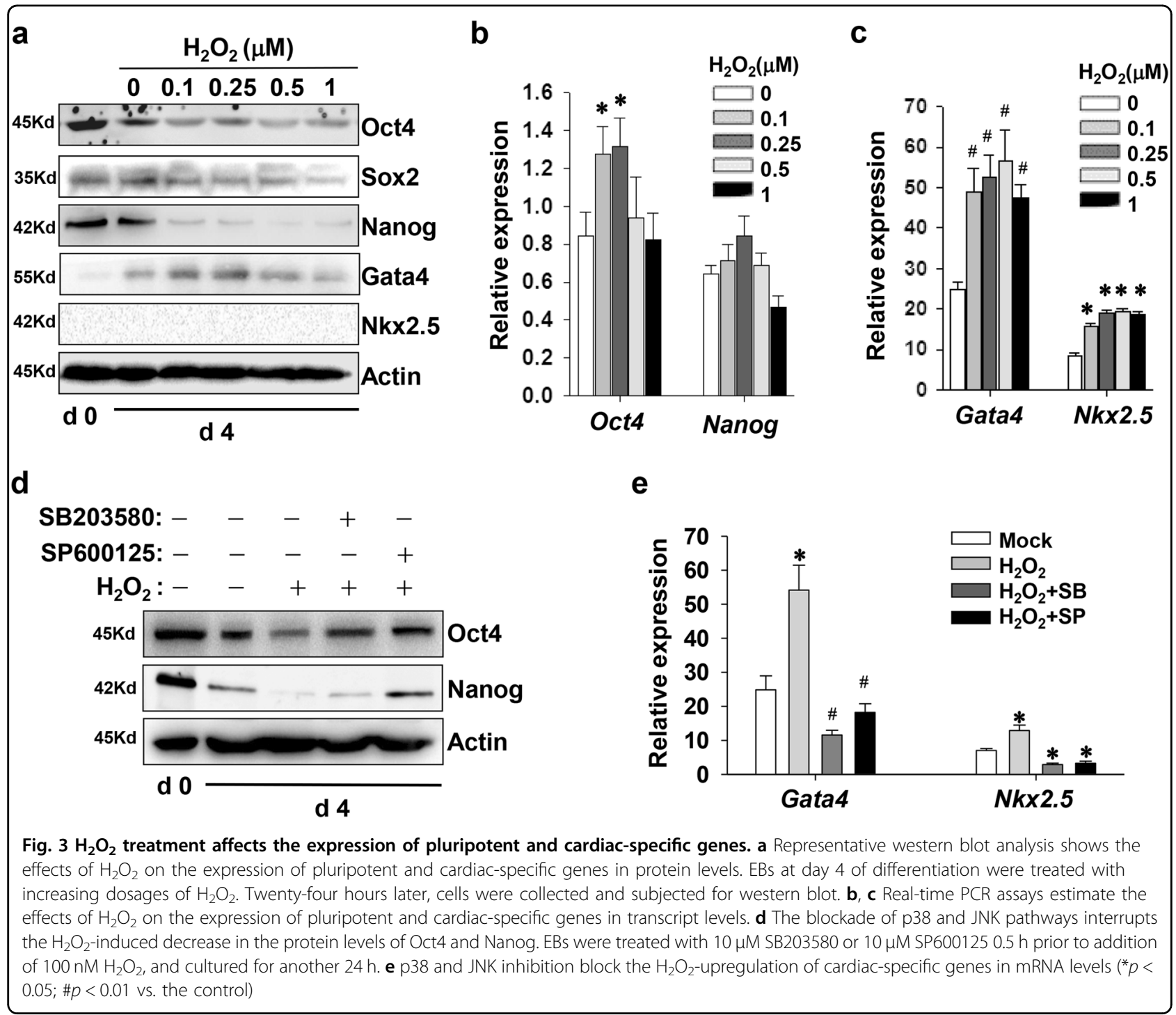

caspase- 3 depletes Hdac4 to activate cardiac differentiation. Firstly, we validated that the expression of Hdac4 gradually decreased in response to increasing amount of $\mathrm{H}_{2} \mathrm{O}_{2}$ (Fig. 6a). As expected, Rapa and TM also triggered the degradation of Hdac4 protein (Fig. 6b). Antagonism of caspase activity indeed reversed the inducible degradation of Hdac4 (Fig. 6c). Furthermore, we performed transient transfection with FLAG-Hdac4 plasmids and found that Hdac4 overexpression indeed reduced the upregulation of Gata4 mRNA during cardiac induction (Fig. 6d, e). Likewise, the transcriptional level of $N k x 2.5$ gene was also abrogated by the forced expression of Hdac4 (Fig. 6e). Obviously, Hdac4 degradation was beneficial for the transactivation of Gata4 and $N k x 2.5$ genes. As a result of Hdac4 depletion, $\mathrm{H}_{2} \mathrm{O}_{2}$ administration increased the levels of H3K9 acetylation on Gata4 and Nkx2.5 promoters. The interference of Z-VAD-FMK dramatically blemished the acetylation status of Gata4 and $N k \times 2.5$ promoters (Fig. 6f). These results further implicate that the expression of cardiac progenitor genes during cardiomyogenesis is tightly linked with Hdac4 downregulation and histone hyper-acetylation at promoter regions.

\section{Nanog cooperates with Hdac4 to inhibit Gata4 and Nkx2.5 expression}

Given the similar roles of Nanog and Hdac4 in regulating cardiac-specific genes, we hypothesized there might be a functional interaction between these two proteins. Subsequently, we performed Co-IP studies in HEK293T cells cotransfected with Nanog and FLAGHdac4 plasmids. As shown in Fig. 7a, Nanog was coimmunoprecipitated with FLAG-Hdac4, suggesting a direct interaction between these two proteins. Meanwhile, immunofluorescence staining showed that Nanog and 
a

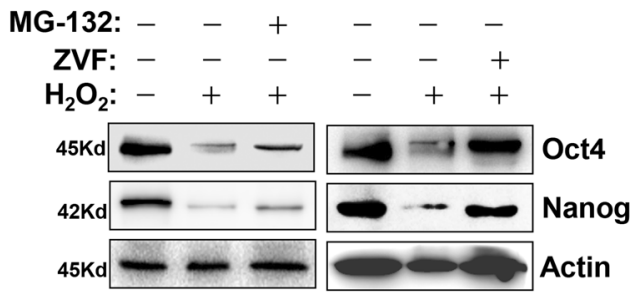

C

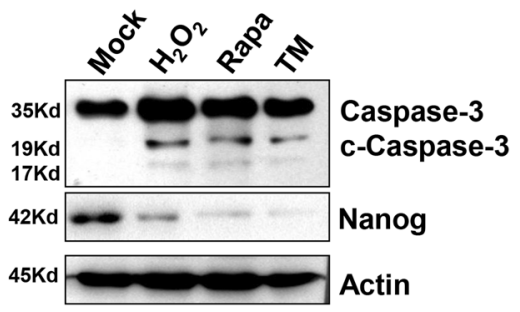

b

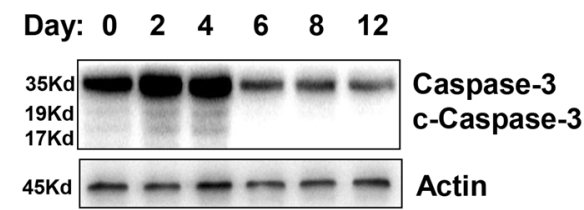

d

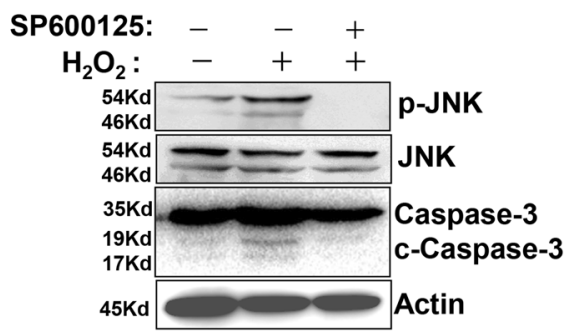

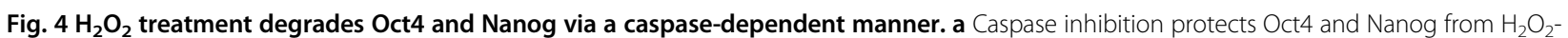
induced degradation. EBs at day 4 of differentiation were treated with $20 \mu \mathrm{M}$ Z-VAD-FMK or $200 \mathrm{nM} \mathrm{MG}-1320.5 \mathrm{~h}$ prior to addition of $100 \mathrm{nM} \mathrm{H} \mathrm{O}_{2}$. Twenty-four hours later, cells were collected and subjected for western blot. $\mathbf{b}$ Representative western blot analysis shows the activation profile of caspase-3 during the whole process of cardiac differentiation. $\mathbf{c}$ EBs at day 4 of differentiation were treated with $\mathrm{H}_{2} \mathrm{O}_{2}$, Rapa, and TM. Two hours later, cells were collected and subjected for western blot to detect the cleavage of caspase-3. d The pretreatment of JNK inhibitor SP600125 decreased $\mathrm{H}_{2} \mathrm{O}_{2}$-induced cleavage of caspase-3

Hdac4 both mainly localized in the nucleus of undifferentiated cells (Fig. 7b). In parallel to an enhanced occupation of Nanog onto the promoters of Gata4 and $N k x 2.5$ genes after Nanog overexpression, an augmented amount of Hdac4 protein was also recruited onto the same genomic regions (Fig. 7c, d). It implicates that Nanog can recruit Hdac4 onto Gata4 and $N k x 2.5$ promoters to establish a silent transcriptional status. Taken together with previous findings, our results suggest that there is a functional association of Nanog and Hdac4 in regulating Gata4 and Nkx2.5 expression.

\section{Excessive ROS promotes Gata4 degradation via JNK cascade}

Herein, we further explored the mechanisms underlying $\mathrm{H}_{2} \mathrm{O}_{2}$-induced Gata4 degradation. Firstly, we found that antagonism of JNK cascade but not p38 reversed the downregulation of Gata4 protein at the presence of 1000 $n M \mathrm{H}_{2} \mathrm{O}_{2}$ (Fig. 8a). Next, we measured the half-life of Gata4 protein after combined transfection with JNK2 plasmid in P19 cells. After transfection, cells were treated with the protein synthesis inhibitor cycloheximide (CHX) and subjected to lysis at indicated time points for the detection of Gata4 degradation. As shown in Fig. 8b, JNK2 overexpression accelerated the degradation of Gata4. Consistent with this result, the proteasome inhibitor MG132 also protected Gata4 protein from $\mathrm{H}_{2} \mathrm{O}_{2}$-induced degradation (Fig. 8c). It implicates that JNK cascade degrades Gata4 protein in a proteasome-dependent manner. Furthermore, we evaluated the effect of JNK2 on Gata4 ubiquitination. As shown in Fig. 8d, forced expression of JNK2 greatly evoked Gata4 ubiquitination compared with vector control. Taken together, our findings indicate that Gata4 is a sensitive factor in response to varying intensity of oxidative stress.

\section{Discussion}

In this study, we revealed the dose-associated effects of ROS on cardiomyogenesis. Mild magnitude of stressproducing stimuli can deplete Nanog and Hdac4 to activate cardiac gene program. However, severe stimuli can degrade Gata4 to interrupt cardiac differentiation.

ROS can act as signaling molecules that extensively participate in the modulation of various cellular events. The effects of ROS are dependent on its intensity and cellular context. In cardiac differentiation, endogenous ROS exerts an indispensable effect. As ROS scavengers such as $\mathrm{N}$-acetyl-cysteine (NAC), catalase, trolox, and pyrrolidine dithiocarbamate, all interrupted the competency of ES cells acquiring cardiac commitment ${ }^{4-6}$. In this study, three stress-producing stimuli, $\mathrm{H}_{2} \mathrm{O}_{2}$, autophagy inducer rapamycin, and ER stress inducer tunicamycin were found to activate cardiomyogenesis via ROS pathway. Relatively, $\mathrm{H}_{2} \mathrm{O}_{2}$ administration at the early stage of differentiation can produce more prominent effects than that at the late stage. The formation stage of EBs is a 


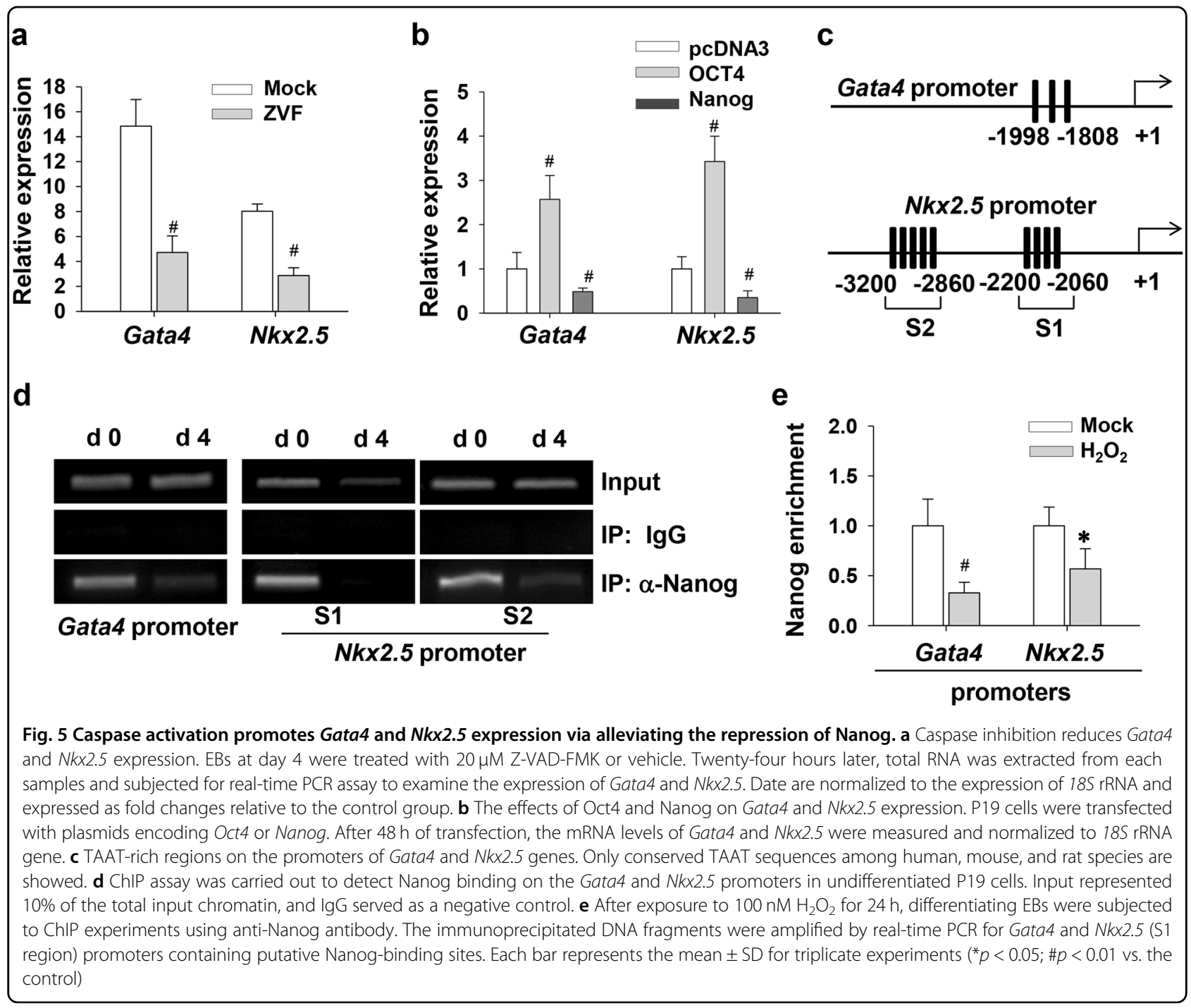

sensitive period for receiving ROS signals and modifying cell fates. Intriguingly, the expression of Nox4 reaches the peak at the late maturation stage of cardiac differentiation. Thus, the redox and differentiation of stem cells is more important in mediating the effects of ROS.

It was well studied that ROS induced the expression of cardiac-specific genes and transcription factors Gata4, $N k x 2.5, M e f 2 C$, as well as differentiation-promoting cytokine $B m p-10^{6}$. Indeed, a previous study has indicated that ROS could trigger the redox activation of c-Jun to upregulate $\mathrm{Gata}^{5}$. Another study showed that ROS evoked p38 activation and nuclear translocation of Mef2 $\mathrm{C}^{4}$. Moreover, ROS is found to induce the activation of PI3K and Jak/STAT and the nuclear translocation of $\mathrm{NF}-\mathrm{\kappa B}$, thereby favoring cardiac differentiation ${ }^{7,14}$. Despite that, it is difficult to explain why the temporal treatment of $\mathrm{H}_{2} \mathrm{O}_{2}$ can generate a long-lasting influence on cardiac gene program. In this study, we found that
$\mathrm{H}_{2} \mathrm{O}_{2}$ treatment can deplete Nanog and Hdac4, hence liberate the repression on Gata4 and $N k x 2.5$ transcription. Histone deacetylation is a repressive modification indicative of gene silence, and histone acetylation is frequently associated with active transcription. Given that Hdac4 alters the epigenetic status of target genes via deacetylating histones, Hdac4 depletion may persistently affect gene expression. Therefore, our results established a link of $\mathrm{H}_{2} \mathrm{O}_{2}$ treatment with the stable and heritable alterations on gene expression in cardiomyogenesis.

Notably, we found that active caspase-3 was required for the ROS-mediated effects. Two inhibitory factors for cardiac differentiation, Nanog and Hdac4, were eliminated via caspase-dependent pathway. The spatiotemporal activation of caspases takes responsibility to eliminate a limited number of selected substrates, thereby reconstructing cell differentiation status. For instance, caspase- 3 cleaves and activates rho-associated kinase-1 


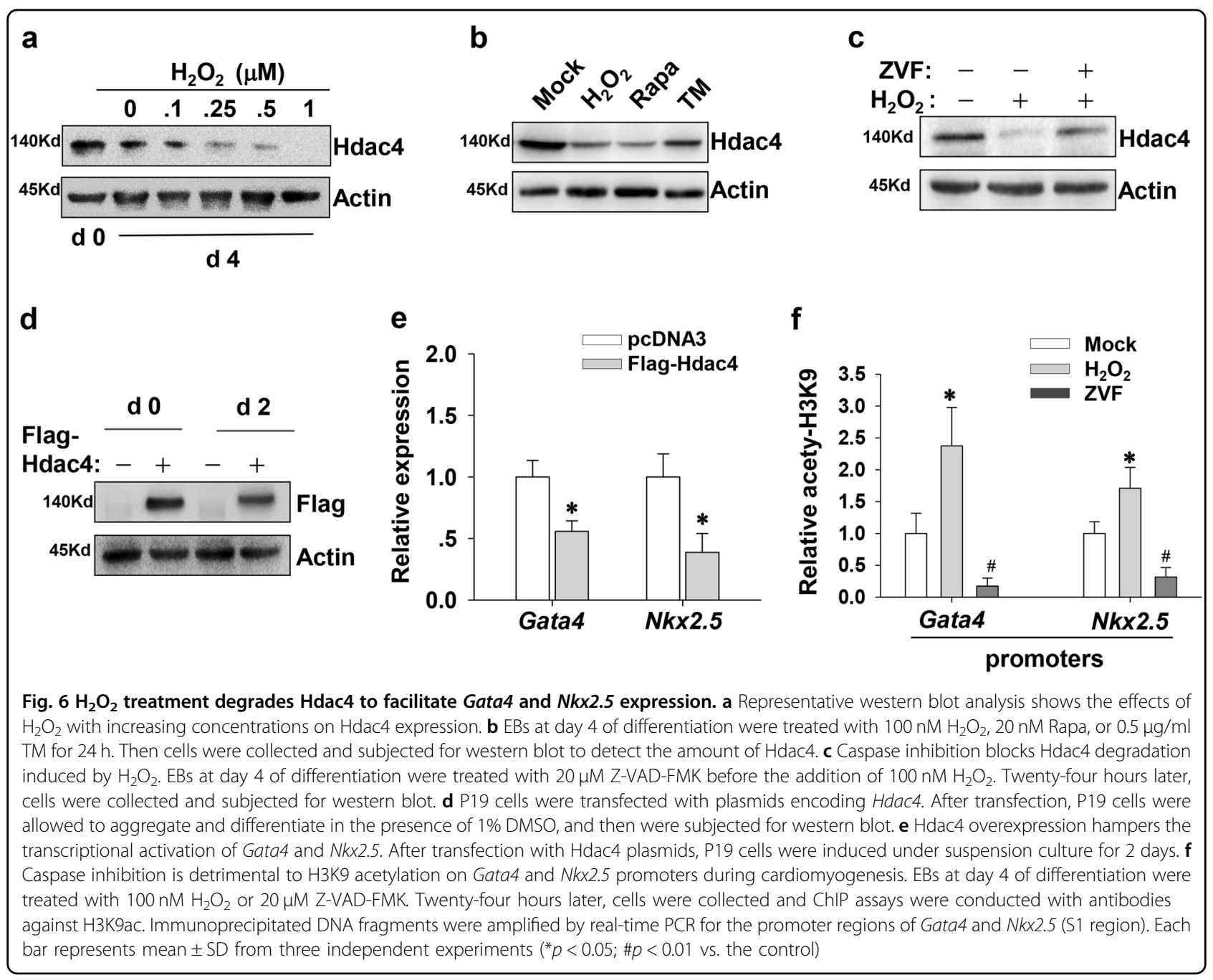

(ROCK-1), and consequently triggers cytoskeletal rearrangement, which is indispensable for macrophage polarity and dendrite pruning during neurogenesis ${ }^{15,16}$. Indeed, previous studies definitely indicated that there exists a temporary activation of caspase- 3 to deplete Nanog when ES cells entering differentiation status from renewal one ${ }^{17,18}$. In this study, our results showed that the spontaneous activation of caspase- 3 occurred at the early stage of cardiac differentiation. Although ROS-activated p38 and JNK kinases enhanced the activation of caspase3 , the driving force of caspase- 3 activation seemed to not be p38 or JNK cascades. Because p38 and JNK kinases reach the optimal activities at the late differentiation stage, and at the same time the cleavage of caspase- 3 disappears reversely. Thus, we convinced that caspase- 3 activation after the onset of cell differentiation is helpful for cell exit from the pluripotent and renewal status, but the trigger mechanism of caspase- 3 activation remains obscure. Even so, the activation of caspase- 3 at the early stage might lay a foundation for the action of ROS stimulation as the agreement of both in time-window.

A core set of transcription factors cooperates together to maintain the pluripotent state of stem cells. The triumvirate of Oct4, Sox2, and Nanog, predispose the expression of genes governing self-renewal and simultaneously repress genes driving differentiation. In this study, we found that Nanog rather than Oct4 plays a crucial role in establishing the transcriptional silence of Gata4 and Nkx2.5. It has been found that Gata 4 can impair the reprogramming transition of fibroblasts from the initiation phase to the iPS status via repressing Nanog expression $^{19}$. Herein, our results suggested that Nanog functionally synergizes with Hdac4 to maintain the transcriptional silence of Gata4 and Nkx2.5 in stem cells. Previous studies showed that Hdac4 inhibition induce the entry of mesodermal cells into the cardiac muscle lineage, companied by the upregulation of Gata4 and $N k \times 2.5$ transcripts $^{20,21}$. It was found that Hdac4 inhibition 


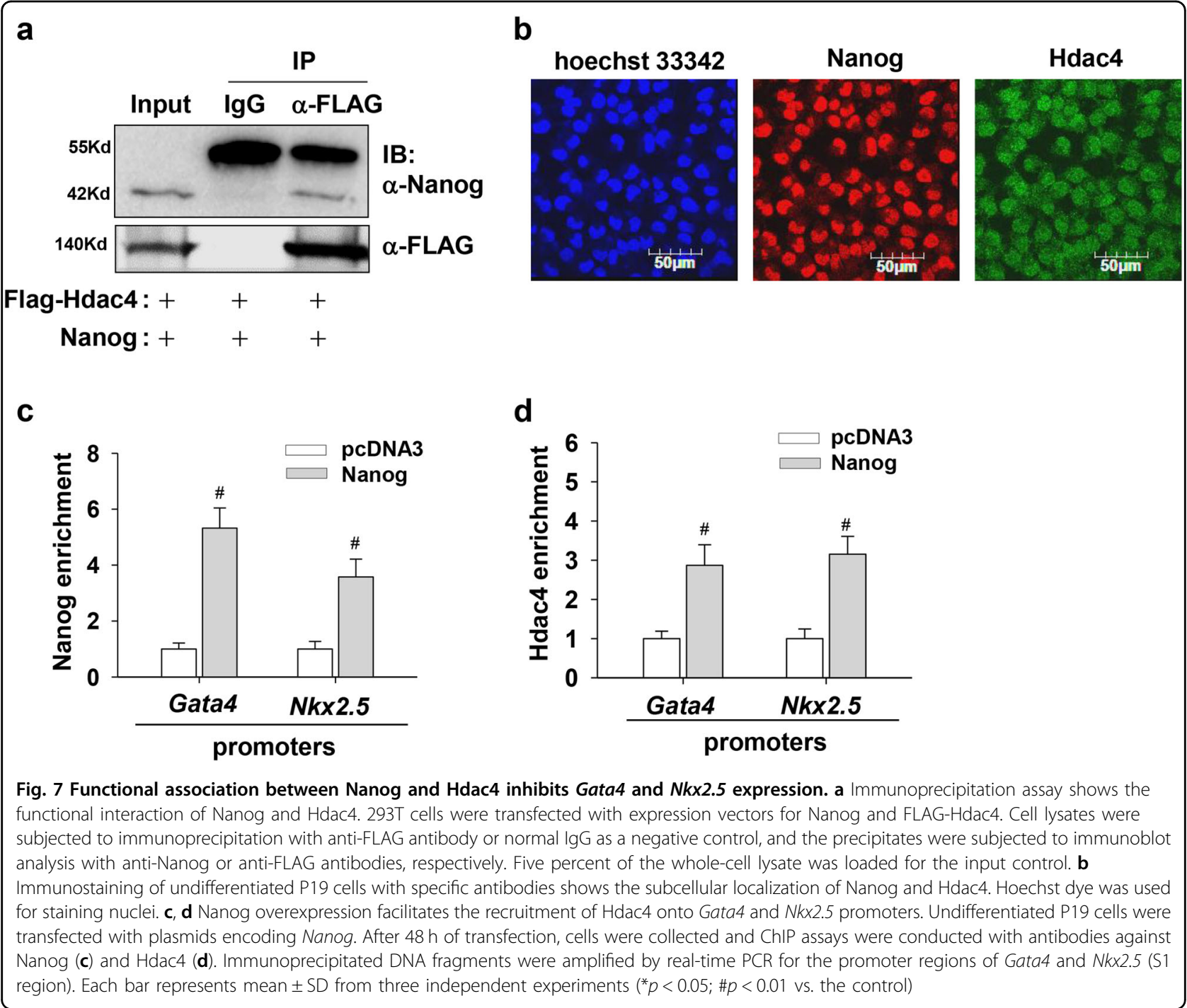

facilitated myocardial differentiation of progenitor cells in infarcted hearts and ameliorated the restoration of cardiac function ${ }^{22}$. Meanwhile, Hdac4 is also sensitive to caspase-3-mediated cleavage just like Nanog. By comparison, it was found that caspase- 3 hardly cleaves Hdac1, 2, 3, 5, and $6^{12,13}$. Based on the similar feature and function of Nanog and Hdac4, we speculated that Hdac4 is a cofactor of Nanog. Herein, our results showed that Nanog can recruit Hdac4 onto the promoters of Gata4 and $N k x 2.5$ to silence their expression. However, the mechanism of functional collaboration between Nanog and Hdac4 is still poorly understood and need more investigation.

Overall, this study reveals that oxidative stress can deplete Nanog and Hdac4 via a caspase-dependent manner, thereby alleviating the repression on Gata4 and Nkx2.5 promoters and favoring cardiac commitment (Fig. 8e). Meanwhile, our study also indicates that intensive stress can activate JNK cascade to degrade Gata4 protein in a proteasome-dependent manner. Thus, stressproducing stimuli exert biphasic and antagonistic effects on cardiac differentiation dependent on its intensity and duration.

\section{Materials and methods}

\section{Cell culture and cardiac differentiation}

P19 embryonal carcinoma cells were cultured in Dulbecco's Modified Eagle Medium (DMEM) containing 15\% heatinactivated Fetal Bovine Serum (FBS) (Hyclone, Waltham, MA, USA), 1\% non-essential amino-acids (Invitrogen, San Diego, CA USA), $1 \mathrm{mM}$ pyruvate, $2 \mathrm{mM}$ Glutamine, $0.1 \mathrm{mM}$ $\beta$-mercaptoethanol in a humidified $5 \% \mathrm{CO}_{2}$ atmosphere at $37^{\circ} \mathrm{C}$. P19 cells $\left(5 \times 10^{5}\right.$ cells $\left./ \mathrm{ml}\right)$ were placed into $60-\mathrm{mm}$ bacterial grade plastic dishes in the presence of $1 \%$ DMSO (Sigma-Aldrich, St. Louis, MO, USA) to initiate cell aggregate and cardiac differentiation. The time point for forming 


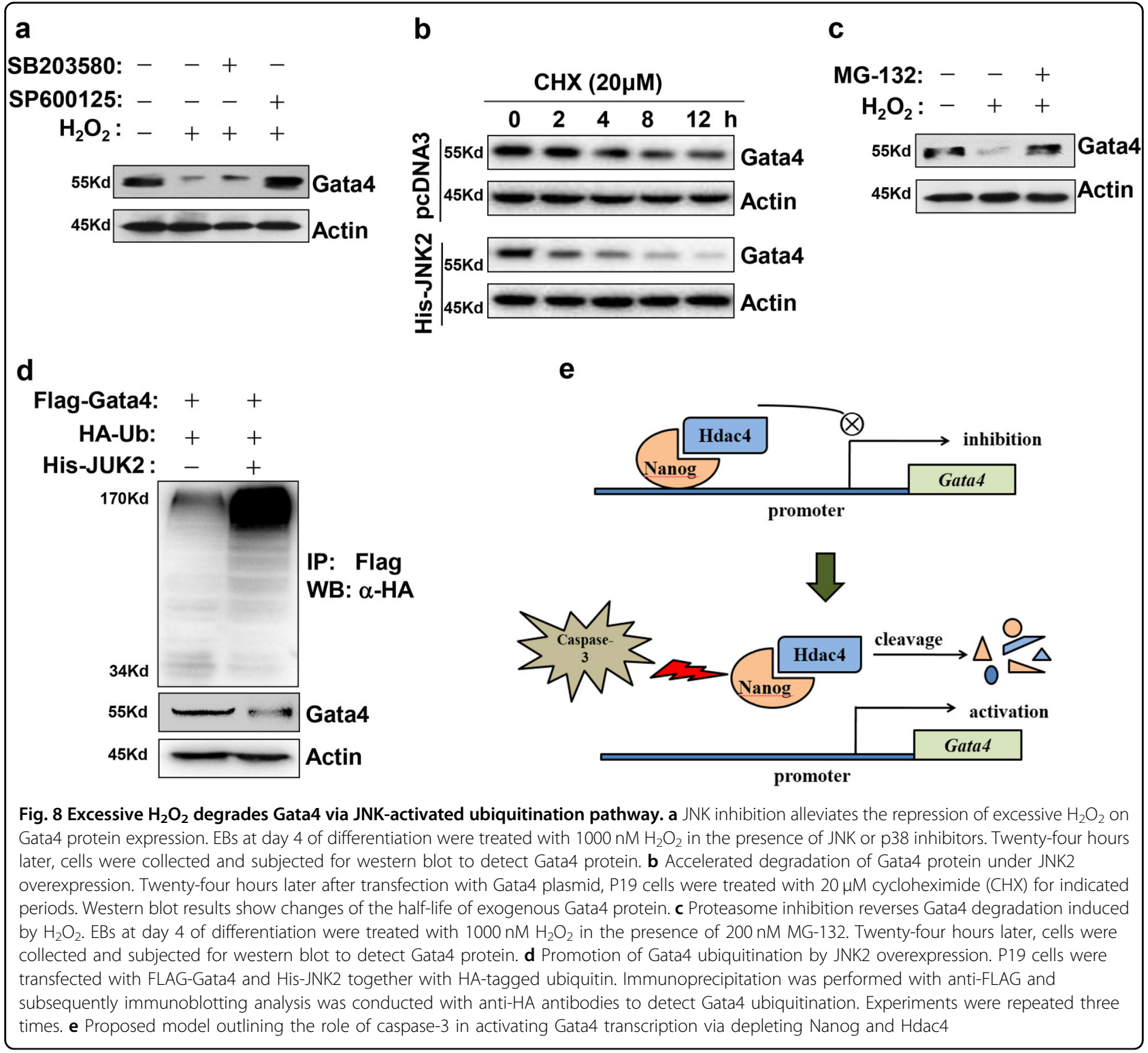

EBs was taken as day 0. At day 4 of cardiac induction, EBs were plated on tissue culture-grade dishes for adherent culture. Spontaneous contractions of EBs were observed from day 8 and the ratio of EBs with beating to the total number of plated EBs was counted.

Total RNA was extracted using Trizol reagent (Invitrogen, San Diego, CA USA). Two micrograms of total RNA from each sample was used for reverse transcription, the PCR was carried out as previously descripted ${ }^{23}$. For real-time reverse transcription polymerase chain reaction (RT-PCR), SYBR Green real-time Master Mix (Toyobo, Japan) and the ABI 7900 Real-Time PCR were used (Applied Biosystems, Foster City, CA, USA). Target gene expression levels were normalized by calculating the Targets/18S expression ratio $(2-\Delta \Delta \mathrm{Ct})$. Primers for RT-
PCR and real-time RT-PCR are listed in the supplemental information, Table S1.

\section{Western blot}

Western blotting has been descripted as previously reported $^{23}$. Antibodies against Oct4, Sox2, Nanog, Gata4, Nkx2.5, Hdac4, and actin were obtained from Santa Cruz Biotechnology (Dallas, TX, USA); caspase-3, p-p38, p38, and JNK were purchased from Cell Signaling Technology (Danvers, MA, USA); FLAG, HA, and $\alpha$-actinin antibodies were from Sigma-Aldrich (Sigma-Aldrich, St. Louis, MO, USA). Antibody specific for the sarcomeric MHC (MF-20; Developmental Studies Hybridoma Bank) was generously provided by Dr. Qin Lu (Key Laboratory of Molecular Cardiovascular Sciences, Peking University, China). The 
figures shown are representative of at least three independent experiments.

\section{Immunofluorescence}

For immunofluorescence identification of differentiated cardiomyocytes, EBs induced until day 12 were digested and replaced on glass coverslips as previously reported ${ }^{23}$. The percentage of $\alpha$-actinin-positive cells out of the total number of cells counted represented the differentiation efficiency. To display the subcellular localization of Nanog and Hdac4, undifferentiated P19 cells were stained with primary antibody against these two proteins, followed by overnight incubation with goat anti-rabbit FITC and donkey anti-mouse TRITC fluorescence-conjugated secondary antibodies (Santa Cruz, Dallas, TX, USA).

\section{Plasmids transfection}

P19 cells were seeded into 12-well plates $24 \mathrm{~h}$ before transfection. Constructs noted in figure legends were transfected using Lipofectamine 2000 (Invitrogen, San Diego, CA USA) according to the manufacturer's instruction. The plasmid encoding Oct4, Nanog, and FLAG-Hdac4 were kindly provided by Dr. Yinan Liu (Stem Cell Research Center, Peking University Health Science Center). His-JNK2 plasmid was purchased from Sino Biological (Beijing, China). After $24 \mathrm{~h}$ of transfection, the cells were digested into single cells and then cultured in suspension to induce cardiac differentiation with $1 \%$ DMSO. All experiments were done in triplicates and performed at least three times.

\section{Chromatin immunoprecipitation (ChIP) assays}

ChIP assays were performed on these cells using the EZ ChIP kit (Millipore, Los Angeles, CA, USA) according to the manufacturer's instructions. Briefly, alter cells were collected and the cross-linked chromatin was sheared to an average DNA fragment length of 100-800 bps, immunoprecipitation was performed using $5 \mu \mathrm{g}$ of Nanog, Hdac4, or acetyl-histone H3K9 antibodies. Normal IgG was used as a negative control. The precipitated DNA was amplified by RT-PCR or real-time PCR. Primers used to amplify the promoters of target genes as following: Gata4 F: CTAAATGCCCAATTCCAG, R: CGACACTTCAGTCC CTCA; Nkx2.5 (S1 region) F: TCAACTCTGGAAGCCCT TAT, R: AGGGTCCTGGGAGTCCTGTT; $N k x 2.5$ (S2 region) F: AGCCAGACGAAGAGCAGA-3, R: AGACAG GCAGCGTTATCC.

\section{Immunoprecipitation}

Briefly, P19 or HEK293T cells were cultured in DMEM containing 15\% heat-inactivated FBS (Hyclone, Waltham, MA, USA), after transfection cells were lysed in modified RIPA buffer for $30 \mathrm{~min}$ at $4{ }^{\circ} \mathrm{C}$ with rotation. Twohundred milligram of cell lysate was pre-cleared with
Protein G-Sepharose (Roche, Indianapolis, IN, USA) for 1 $h$, and then was incubated with anti-FLAG conjugated agarose beads (Sigma-Aldrich, St. Louis, MO, USA) overnight at $4{ }^{\circ} \mathrm{C}$ with rotation. Agarose beads were washed with TBS buffer and denatured samples were separated by sodium dodecyl sulfate polyacrylamide gel electrophoresis electrophoresis. Western blot was performed as described above.

\section{Flow cytometry}

The $\alpha$-actinin and the rabbit IgG isotype control antibodies were purchased from Sigma (Sigma-Aldrich, Pudong, Shanghai, China), the secondary FITC conjugated secondary antibody was purchased from Invitrogen (ThermoFisher Scientific, Carlsbad, CA, USA). Briefly, P19 cells were induced to beating cardiomyocytes and treated as designed, then trypsined to solo suspension cells, after Fc blocking cells were incubated with primary or secondary antibodies in 3\% BSA/PBS for $30 \mathrm{~min}$ at room temperature in the dark, finally cells were washed three times and resuspended in $500 \mu \mathrm{l}$ cold PBS and analyzed using a FACS Calibur flow cytometer (BD, Franklin Lakes, NJ, USA).

\section{Statistical analysis}

All data were expressed as the mean \pm SD from at least three independent biological replicates. The unpaired Student's $t$-test or one way ANOVA with significance set at $p<0.05$ was used to determine statistical significance for each assay. Error bars indicate standard deviation.

\section{Acknowledgements}

This work was supported by the National Natural Science Foundation of China (31470082, 81101930, T.L.; 81670201 , Z.L.), Zhejiang Provincial Natural Science Foundation and Public Innovation Program (LY14C120001, 2014C37126, T.L.); the Talent Program of Tianjin Medical University (11601501/2016KJ0317, Z.L.), the First-Class Disciplines "Basic Science and Clinical Research of Cardiovascular and Metabolic Diseases" Program of Tianjin Medical University (2016XK010303, Z.L.), and the Thousand Talents Plan of Tianjin (11601501/2016DW0202, Z.L.).

\section{Author details}

'School of Medicine, Hunan Normal University, Changsha, Hunan 410013, China. ${ }^{2}$ Department of Biology, Zhejiang Normal University, Jinhua, Zhejiang 321004, China. ${ }^{3}$ Department of Physiology and Pathophysiology, School of

Basic Medical Science, Tianjin Medical University, Heping, Tianjin 300070, China

Conflict of interest

The authors declare that they have no conflict of interest.

Publisher's note

Springer Nature remains neutral with regard to jurisdictional claims in published maps and institutional affiliations.

Supplementary Information accompanies this paper at (https://doi.org/ 10.1038/s41419-018-0281-y).

Received: 12 October 2017 Revised: 25 December 2017 Accepted: 28 December 2017 
Published online: 14 February 2018

\section{References}

1. Parikh, A., Wu, J. C., Blanton, R. M. \& Tzanakakis, E. S. Signaling pathways and gene regulatory networks in cardiomyocyte differentiation. Tissue Eng. Part BRe 21, 377-392 (2015)

2. Kattman, S. J. et al. Stage-specific optimization of activin/nodal and BMP signaling promotes cardiac differentiation of mouse and human pluripotent stem cell lines. Cell Stem Cell 8, 228-240 (2011).

3. Lian, X. J. et al. Robust cardiomyocyte differentiation from human pluripotent stem cells via temporal modulation of canonical Wnt signaling. Proc Natl. Acad. Sci. USA 109, E1848-E1857 (2012).

4. Li, J. et al. The NADPH oxidase NOX4 drives cardiac differentiation: Role in regulating cardiac transcription factors and MAP kinase activation. Mol. Biol. Cell 17, 3978-3988 (2006).

5. Murray, T. V. A., Smyrnias, I., Shah, A. M. \& Brewer, A. C. NADPH oxidase 4 regulates cardiomyocyte differentiation via redox activation of C-Jun protein and the cis-regulation of GATA-4 gene transcription. J. Biol. Chem. 288, 15745-15759 (2013).

6. Buggisch, M. et al. Stimulation of ES-cell-derived cardiomyogenesis and neonatal cardiac cell proliferation by reactive oxygen species and NADPH oxidase. J. Cell Sci. 120, 885-894 (2007). (Pt 5).

7. Sauer, H., Rahimi, G., Hescheler, J. \& Wartenberg, M. Role of reactive oxygen species and phosphatidylinositol 3-kinase in cardiomyocyte differentiation of embryonic stem cells. FEBS Lett. 476, 218-223 (2000).

8. Crespo, F. L., Sobrado, V. R., Gomez, L., Cervera, A. M. \& McCreath, K. J. Mitochondrial reactive oxygen species mediate cardiomyocyte formation from embryonic stem cells in high glucose. Stem Cells 28, 1132-1142 (2010).

9. Heo, J. S. \& Lee, J. C. beta-Catenin mediates cyclic strain-stimulated cardiomyogenesis in mouse embryonic stem cells through ROS-dependent and integrin-mediated PI3K/Akt pathways. J. Cell. Biochem. 112, 1880-1889 (2011).

10. Osman, B. et al. Rapamycin induces the TGFbeta1/Smad signaling cascade in renal mesangial cells upstream of mTOR. Cell Signal. 21, 1806-1817 (2009).
11. Lim, E. J., Heo, J. \& Kim, Y. H. Tunicamycin promotes apoptosis in leukemia cells through ROS generation and downregulation of survivin expression. Apoptosis 20, 1087-1098 (2015).

12. Paroni, G. et al. Caspase-dependent regulation of histone deacetylase 4 nuclear-cytoplasmic shuttling promotes apoptosis. Mol. Biol. Cell 15, 2804-2818 (2004).

13. Liu, F., Dowling, M., Yang, X. -J. \& Kao, G. D. Caspase-mediated specific cleavage of human histone deacetylase 4. J. Biol. Chem. 279, 34537-34546 (2004).

14. Sauer, H., Rahimi, G., Hescheler, J. \& Wartenberg, M. Effects of electrical fields on cardiomyocyte differentiation of embryonic stem cells. J. Cell. Biochem. $\mathbf{7 5}$ 710-723 (1999)

15. Liu, Y. et al. Mouse macrophage polarity and ROCK1 activity depend on RhoA and non-apoptotic Caspase 3. Exp. Cell Res. 341, 225-236 (2016).

16. Erturk, A., Wang, Y. \& Sheng, M. Local pruning of dendrites and spines by caspase-3-dependent and proteasome-limited mechanisms. J. Neurosci. 34, 1672-1688 (2014)

17. Fujita, J. et al. Caspase activity mediates the differentiation of embryonic stem cells. Cell Stem Cell 2, 595-601 (2008).

18. Akbari-Birgani, S., Hosseinkhani, S., Mollamohamadi, S. \& Baharvand, H. Delay in apoptosome formation attenuates apoptosis in mouse embryonic stem cell differentiation. J. Biol. Chem. 289, 16905-16913 (2014).

19. Serrano, F. et al. Gata4 blocks somatic cell reprogramming by directly repressing Nanog. Stem Cells 31, 71-82 (2013).

20. Karamboulas, C. et al. HDAC activity regulates entry of mesoderm cells into the cardiac muscle lineage. J. Cell Sci. 119, 4305-4314 (2006). (Pt 20).

21. Chen, H. P. et al. HDAC inhibition promotes cardiogenesis and the survival of embryonic stem cells through proteasome-dependent pathway. J. Cell. Biochem. 112, 3246-3255 (2011).

22. Zhang, L. X. et al. Specific inhibition of HDAC4 in cardiac progenitor cells enhances myocardial repairs. Am. J. Physiol. Cell Physiol. 307, C358-C372 (2014).

23. Liu, Z. et al. WNT signaling promotes Nkx2.5 expression and early cardiomyogenesis via downregulation of Hdac1. Biochim. Et. Biophys. Acta 1793, 300-311 (2009). 